EPJ Web of Conferences 70, 00085 (2014)

DOI: $10.1051 /$ epjconf/ 20147000085

(C) Owned by the authors, published by EDP Sciences, 2014

\title{
CLIC: Status and Plans
}

André Sailer ${ }^{1 a}$ on behalf of the CLIC physics and detector study and the CLIC accelerator team ${ }^{1}$ CERN, Geneva, Switzerland

\begin{abstract}
The Compact Linear Collider (CLIC) is a high energy electron-positron collider with a maximal centre-of-mass energy of $3 \mathrm{TeV}$. In order to achieve high luminosity small bunches with high intensity are necessary. These lead to strong beam-beam forces, which create a challenging background environment. The accelerator concept and the detectors designed for CLIC are presented. Results from detector benchmark studies presented in the CLIC conceptual design report are summarised.
\end{abstract}

\section{Introduction}

A high-luminosity and high-energy electron-positron collider allows for precise measurements of Standard Model and Beyond Standard Model physics. The Compact Linear Collider (CLIC) is designed for electron-positron collisions at centre-of-mass energies of up to $3 \mathrm{TeV}$, and is offering a possible physics reach complementary to that of the LHC. In 2012 a Conceptual Design Report (CDR) for the CLIC accelerator, detailing the technological feasibility issues, will be published [1]. The report concentrates on a machine optimised for $3 \mathrm{TeV}$. The preferred energy, from a physics pointof-view, will be determined largely based on the results from the LHC. The feasibility of a detector operating in the conditions of the CLIC collider has been evaluated in a separate CDR volume [2].

\section{CLIC Accelerator}

The CLIC accelerator uses a novel two-beam acceleration scheme. The normal-conducting radiofrequency (RF) cavities operate at a gradient of $100 \mathrm{MV} / \mathrm{m}$. The RF is produced by decelerating a high-current low-energy drive beam. In order to achieve the desired luminosity small bunches with a transverse bunch size of $45 \mathrm{~nm}$ by $1 \mathrm{~nm}$ and a length of $44 \mu \mathrm{m}$ are needed. The normal conducting cavity technology limits the length of the RF pulse, therefore the length of a bunch train and the bunch spacing is favoured to be quite small.

\subsection{CLIC Overview}

Figure 1 shows the layout of the $3 \mathrm{TeV}$ CLIC facility. The $3 \mathrm{TeV}$ machine needs two drive-beam generation complexes, where the drive beams are accelerated and combined into the subtrains for the acceleration of the main beam. The main-beam source provides electron and positron beams with very

a e-mail: andre.philippe.sailer@ cern.ch

This is an Open Access article distributed under the terms of the Creative Commons Attribution License 2.0, which permits unrestricted use, distribution, and reproduction in any medium, provided the original work is properly cited. 


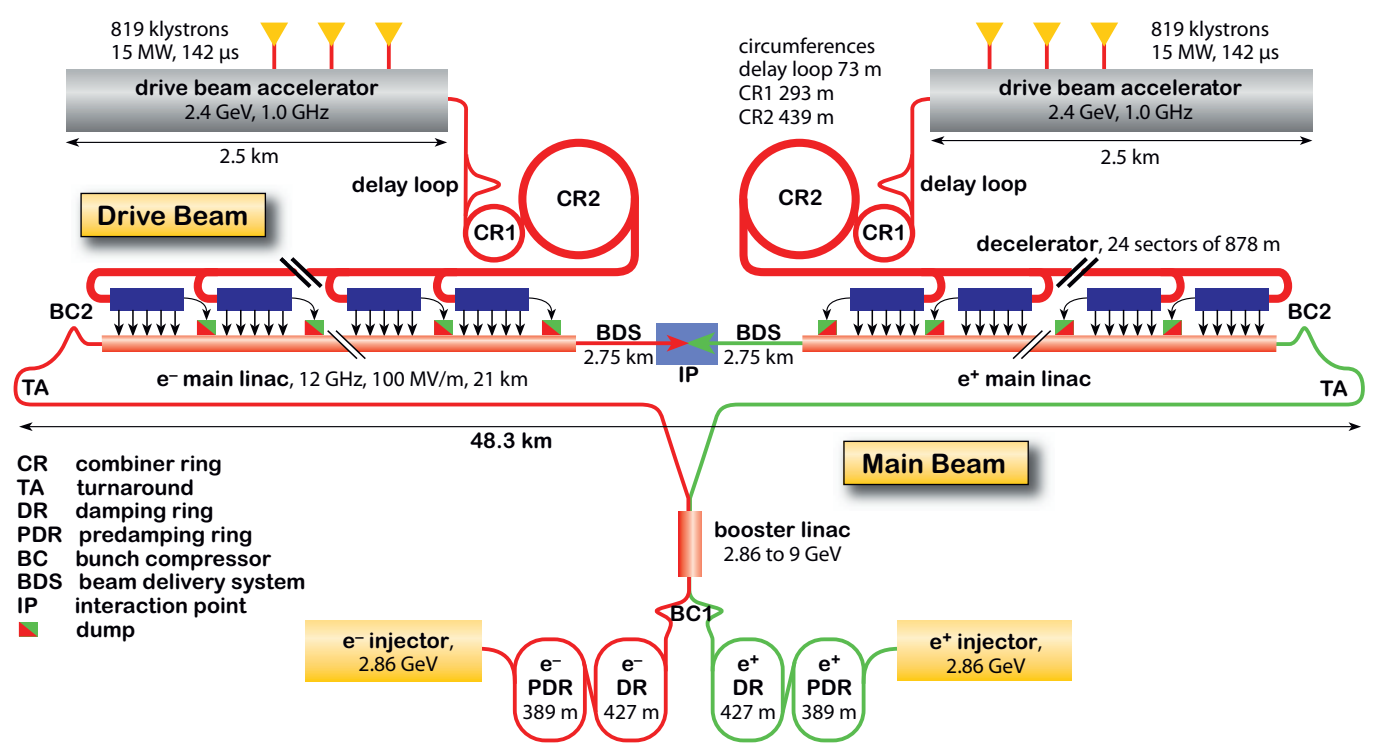

Figure 1. CLIC $3 \mathrm{TeV}$ accelerator layout [3].

small emittances. In the main linear accelerator the drive beams are decelerated and the main beams are accelerated up to the final energy, while maintaining their small emittances. The beam-delivery system prepares the beams for the collision in the interaction region, where two detectors share a single interaction point via a push-pull scheme.

The beam parameters at the interaction point for the $3 \mathrm{TeV}$ CLIC, and the nominal $14 \mathrm{TeV}$ LHC for comparison, are given in Table 1. While aiming at similar luminosities of $10^{34} \mathrm{~cm}^{-2} \mathrm{~s}^{-1}$ the LHC beams can be orders of magnitude larger than the CLIC beams, because the train repetition rate is much larger in the circular collider.

\subsection{Drive Beam Generation}

One of the main feasibility issues for the two-beam acceleration scheme is the efficient generation of a high-current drive beam to provide the $12 \mathrm{GHz}$ RF. The drive beam is accelerated in a $140 \mu$ s long pulse consisting of bunches at a frequency of about $500 \mathrm{MHz}$ ( 2 ns bunch spacing), and subtrains of 244 ns length. After the acceleration to $2.4 \mathrm{GeV}$, every second subtrain is delayed by $244 \mathrm{~ns}$ in the delay loop, which produces subtrains of $244 \mathrm{~ns}$ length with a bunch spacing of $1 \mathrm{~ns}$ followed by a gap of 244 ns (Figure 2 top). The following two combiner rings (CR) decrease the bunch spacing by a factor 12, which results in a drive beam consisting of 24 subtrains of 244 ns length with a bunch spacing of $83 \mathrm{ps}$ (a frequency of $12 \mathrm{GHz}$ ) and a gap between the trains of $5.85 \mu \mathrm{s}$ [1] (see also Figure 2).

One of the drive-beam accelerator and generator complexes for the full CLIC accelerator is shown in Figure 3. It consists of a delay loop and two combiner rings, one for a compression factor of three and the second one with a factor of four. 
ICFP 2012

Table 1. Accelerator parameter for $3 \mathrm{TeV}$ CLIC [1] and $14 \mathrm{TeV}$ LHC [4]

\begin{tabular}{lcc}
\hline & CLIC 3 TeV & LHC $14 \mathrm{TeV}$ \\
\hline Colliding particles & Electron-Positron & Proton-Proton \\
Luminosity $\left[10^{34} / \mathrm{cm}^{2} / \mathrm{s}\right]$ & 5.9 & 1.0 \\
Beam size in $X / Y / Z$ & $45 \mathrm{~nm} / 1 \mathrm{~nm} / 44 \mu \mathrm{m}$ & $16.7 \mu \mathrm{m} / 16.7 \mu \mathrm{m} / 7.55 \mathrm{~cm}$ \\
Bunch charge & $3.72 \cdot 10^{9}$ & $1.15 \cdot 10^{11}$ \\
BX separation & $0.5 \mathrm{~ns}$ & $25 \mathrm{~ns}$ \\
Bunches per train & 312 & 2808 \\
Repetition rate & $50 \mathrm{~Hz}$ & $11.2 \mathrm{kHz}$ \\
\hline
\end{tabular}
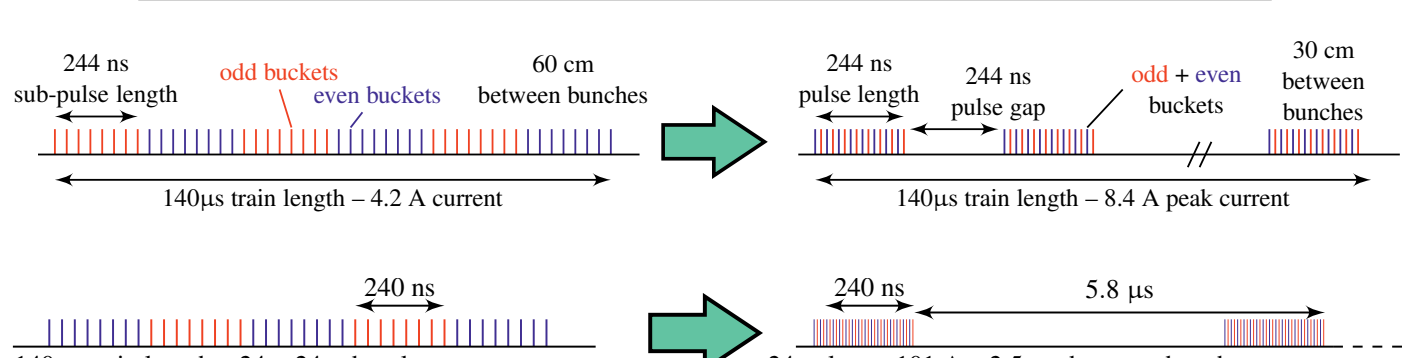

$140 \mu$ s train length $-24 \times 24$ sub-pulses

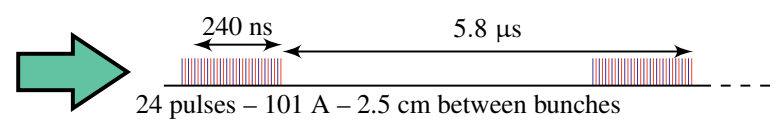

$4.2 \mathrm{~A}-2.4 \mathrm{GeV}-60 \mathrm{~cm}$ between bunches

Figure 2. Bunch structure of the drive beam after acceleration and (top) after the delay loop, and (bottom right) after the combination with delay loop and combiner ring.

The 24 subtrains of the drive beam are transferred to the acceleration sectors (blue rectangles in Figure 1). In the acceleration sectors the drive beam is decelerated to $240 \mathrm{MeV}$ in power extraction structures (PETS), and the RF power is transferred to the acceleration cavities for the main beam.

The drive beam combination with a delay loop and a single combiner ring was achieved in the CLIC Test Facility 3 (CTF3) [1]. Figure 4 shows the current measured at various points in the CTF3 beam line. The beam begins with a current of about $3.5 \mathrm{~A}$; half of the bunches pass through the delay loop, resulting in the gaps of the measured current. After the delay loop the current is 7 A with gaps between the subtrains. Then the bunches reach the combiner ring, where the sub-pulses are joined together for a maximal peak current of about 27.6 A.

\subsection{Two-Beam Acceleration}

Another important feasibility issue concerns the acceleration gradient for the main beam and the break-down rate of the copper cavities and PETS. The two-beam acceleration was successfully performed at the CTF3. Figure 5 shows the energy of the main beam of CTF3 with (top) and without (bottom) RF power in the acceleration module. The RF power was provided by the deceleration of the drive beam in the power extraction structure. The energy difference of $23.08 \mathrm{MeV}$ corresponds to a gradient of $106 \mathrm{MV} / \mathrm{m}$.

The break-down rate of the accelerating cavities and PETS have been studied in klystron based test facilities at SLAC and KEK. The recent generation of cavities and PETS reached a break-down rate below the required probability [1]. 


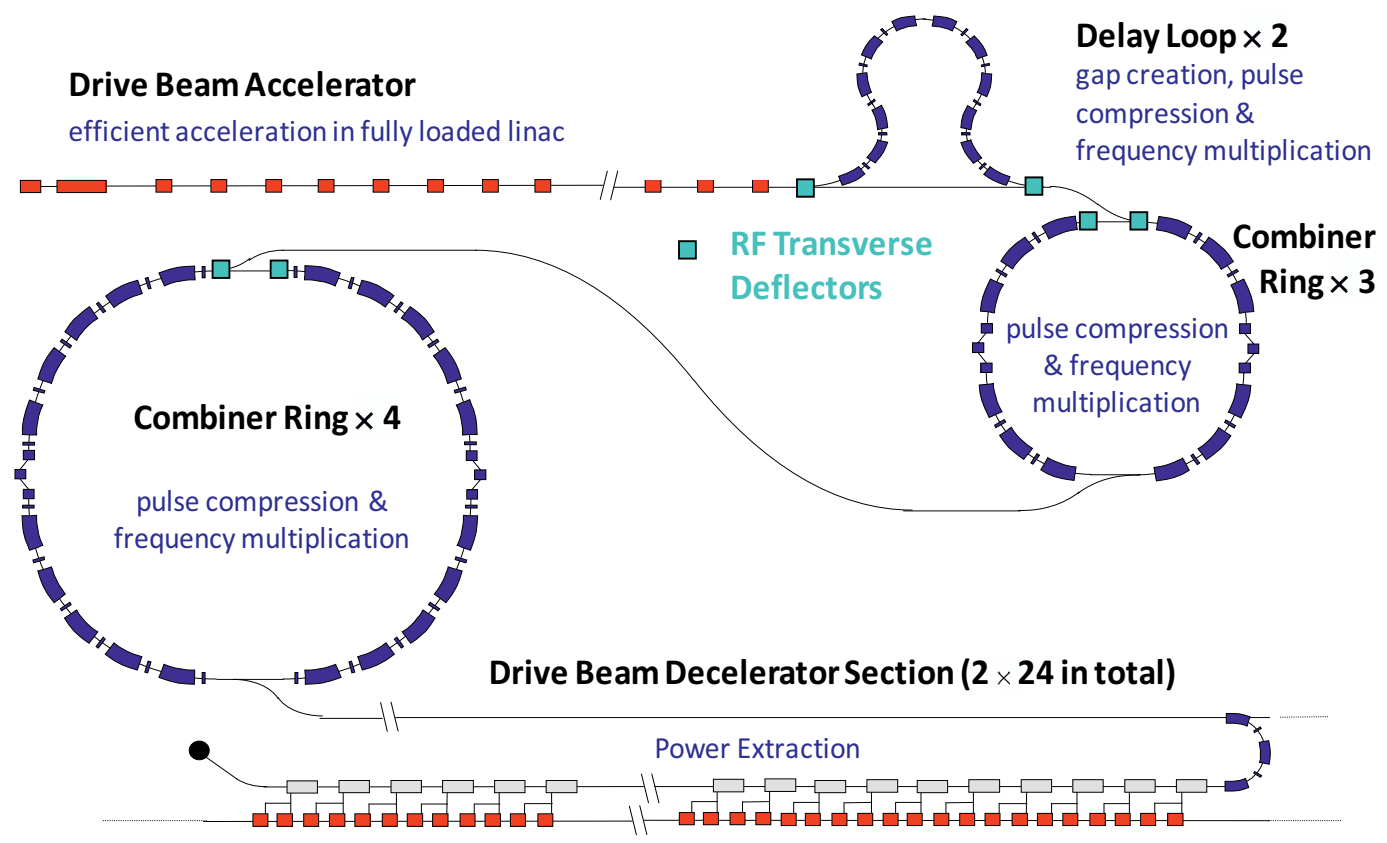

Figure 3. Drive-beam accelerator and generator [1].

\subsection{Accelerator: Conclusions and Outlook}

Key issues have been identified in the accelerator CDR for CLIC, and feasibility was successfully shown in CTF3 and elsewhere. It has been shown that drive beam generation and two-beam acceleration is possible. The newer generations of cavities and PETS fulfil the requirements for the break-down rate. Progress was made on the implementation of a two-beam acceleration based collider. However, more work is needed for CLIC towards a detailed technical design, which will be addressed in the next phase of the CLIC project [3].

\section{Physics and Detectors at CLIC}

The accelerator and detector design studies are closely linked. The short repetition rate of $0.5 \mathrm{~ns}$ between the 312 bunch crossings of a train puts a constraint on the time-stamping capabilities of the detectors, as a single bunch crossing cannot be identified online, but only through offline analysis. The rather slow train repetition rate of $50 \mathrm{~Hz}$ allows for the readout of the complete detector during the $20 \mathrm{~ms}$ between bunch trains, or the electronics can switch off to reduce the power consumption. The feasibility and required technologies of a detector operating under CLIC conditions was studied in the physics and detector CDR [2]. 


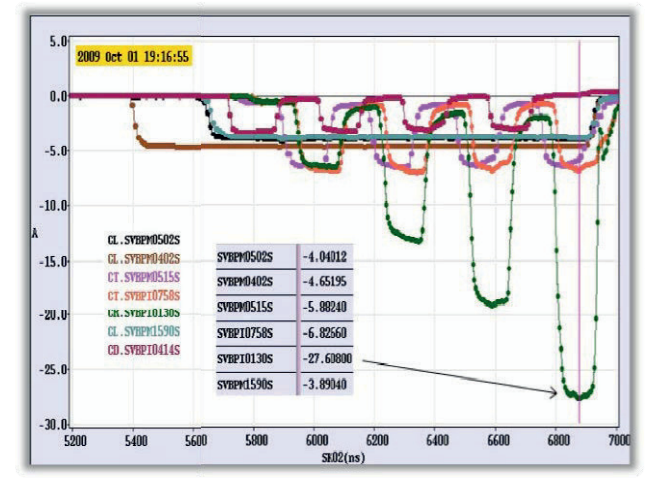

Figure 4. Drive beam accelerator intensity and frequency multiplication by a factor 8 as measured in CTF3 [1].
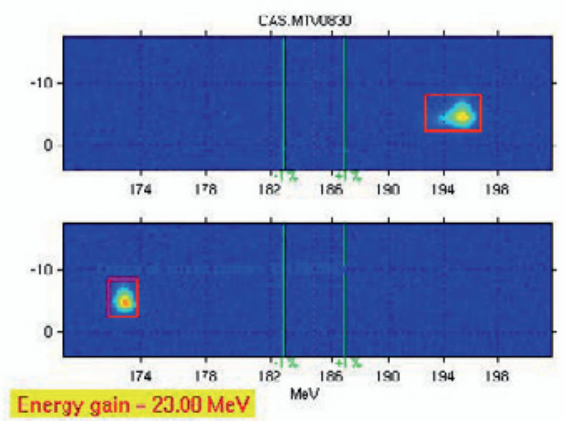

Figure 5. Example acceleration in the two-beam test stand with the $12 \mathrm{GHZ}$ RF power on(top) and off(bottom). The energy gain of $23.08 \mathrm{MeV}$ corresponds to a gradient of $106 \mathrm{MV} / \mathrm{m}$ (from [1]).

\subsection{Detector for CLIC}

The detector requirements for CLIC are driven by precision measurements. The momentum resolution plays a key role in the measurement of the lepton energy-distribution endpoints in the slepton mass measurement [5]. Figure 6 (left) shows the impact of the momentum resolution on the precise determination of the endpoint. The momentum resolution is also important for the measurement of the $\mathrm{h} \rightarrow \mu \mu$ branching ratio, and the model-independent measurements of the Higgs-boson via the Z-recoil, where the Z-boson decays to two muons or electrons. For all of these processes a momentum resolution of $\sigma_{p_{\mathrm{T}}} / p_{\mathrm{T}}^{2}=2 \cdot 10^{-5} \mathrm{GeV}^{-1}$ is required.

The jet-energy resolution has to be good enough to distinguish between jets from the W-, Z- or Higgs-boson, which leads to a jet-energy resolution requirement of $\sigma_{E} / E \approx 5 \%-3.5 \%$ for jet energies of $50 \mathrm{GeV}$ to $1 \mathrm{TeV}$. Figure 6 (right) illustrates the effect of the jet-mass resolution.

For efficient b-tagging (e.g., for Higgs branching ratio measurements) a good impact parameter resolution of $\sigma_{r \phi}=\left(5 \oplus 15 /\left(p[\mathrm{GeV}] \sin ^{\frac{3}{2}} \theta\right)\right) \mu \mathrm{m}$ is required. To reach a multiple-scattering term of about $15 \mu \mathrm{m}$, a material budget of less than $0.2 \%$ radiation lengths per layer of the vertex detector (including support and cooling) is allowed.

The detector also has to provide good lepton identification, angular coverage down to small polar angles, and very forward electron tagging. The detectors used for the benchmark studies are described in references $[6,7]$ and the CDR [2].

\subsection{Beam-Induced Backgrounds}

The small beam sizes necessary to reach the required luminosity and the high energy lead to large electromagnetic fields during the bunch crossings. The strong fields lead to the deflection of the beam particles, which start to radiate a form of synchrotron radiation, called Beamstrahlung [8]. The energy loss due to Beamstrahlung results in the luminosity spectrum. Not all collisions will take place at the nominal centre-of-mass energy. The luminosity spectrum of the $3 \mathrm{TeV}$ CLIC is shown in Figure 7 (left). About $30 \%$ of the events take place above $99 \%$ of the nominal centre-of-mass energy. The remaining luminosity is not lost, as CLIC will probably operate far above the production threshold 

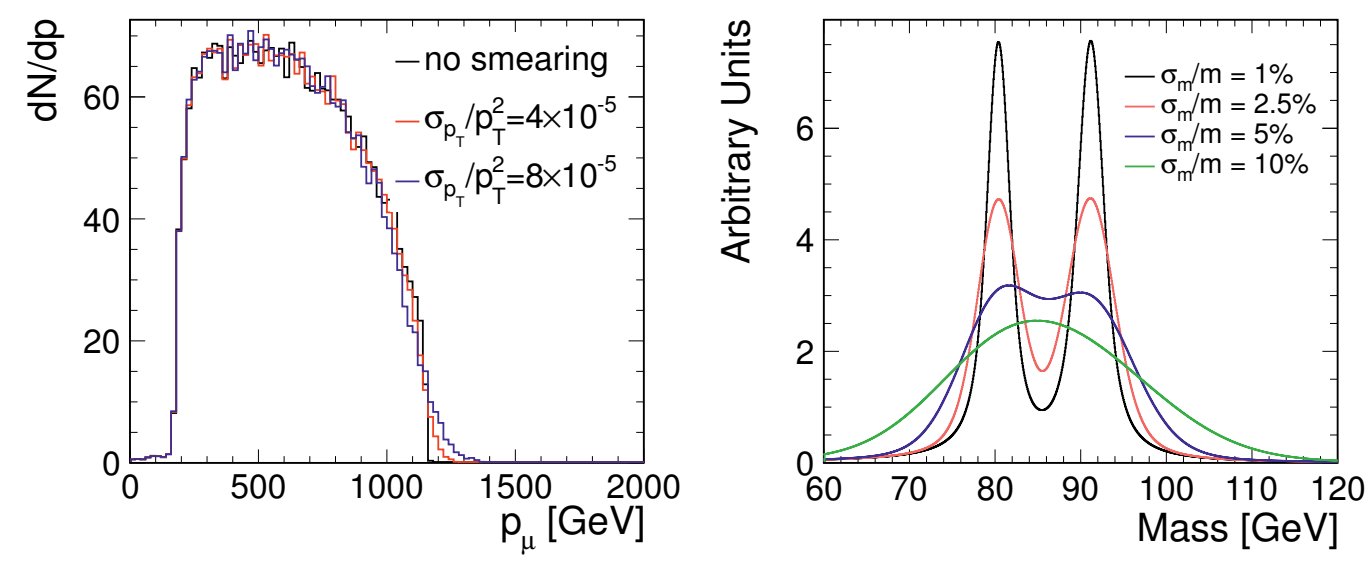

Figure 6. Effect of different resolutions for (left) track momentum reconstruction and (right) jet mass reconstruction [2].
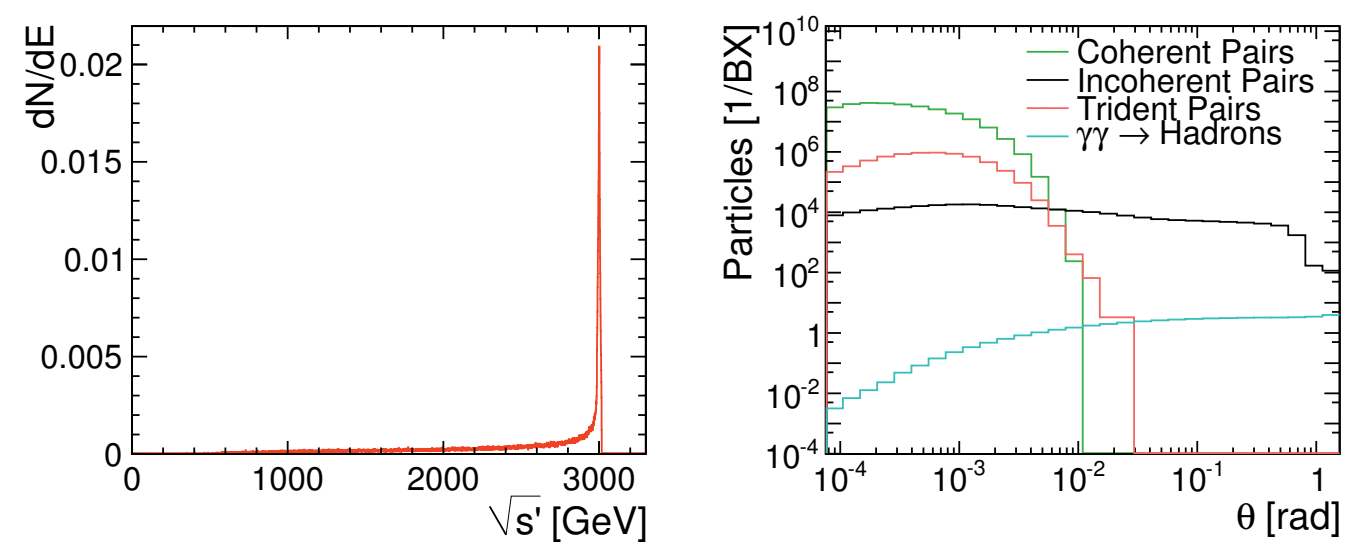

Figure 7. Left: Luminosity spectrum for $3 \mathrm{TeV}$ CLIC. Right: Angular distribution for the particles from different background processes for $3 \mathrm{TeV}$ CLIC [2].

for new particles. For example, the fraction of useful luminosity for a process with a threshold of $2 \mathrm{TeV}$ is greater than $75 \%$.

The beam-beam interactions also lead to a large rate of background particles. The coherent processes [9] are only producing particles at very shallow angles, which can leave the detector without interactions. The incoherently produced electron-positron pairs and the $\gamma \gamma \rightarrow$ hadron events, however, cause a significant background in the detector. Figure 7 (right) shows the angular distribution of the different background processes. The rate of incoherent pairs in the detector is suppressed partially by the strong solenoid field. 

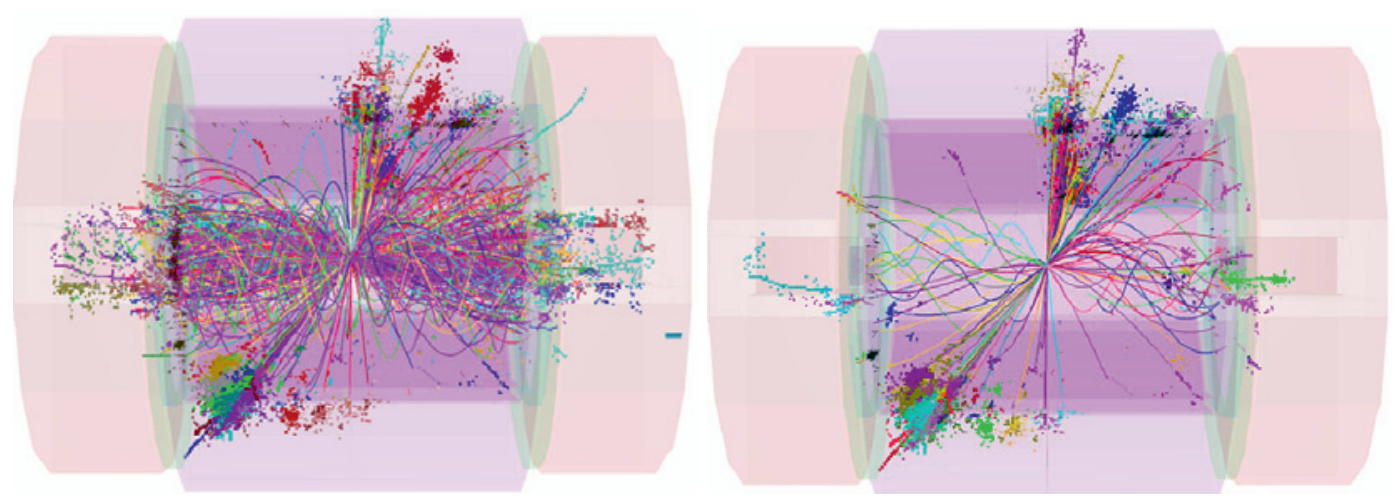

Figure 8. (left) Reconstructed particles in a simulated $\mathrm{e}^{+} \mathrm{e}^{-} \rightarrow \mathrm{H}^{+} \mathrm{H}^{-} \rightarrow \mathrm{t} \overline{\mathrm{b}} \overline{\mathrm{t}}$ event at $3 \mathrm{TeV}$ with background from $\gamma \gamma \rightarrow$ hadrons overlaid. (right) the effect of applying tight timing cuts on the reconstructed cluster times [2].

The $\gamma \gamma \rightarrow$ hadron events show a harder transverse momentum spectrum than the incoherent pairs. These events deposit a large amount of visible energy in the detector. During a bunch train 5000 tracks with a total momentum of $7 \mathrm{TeV}$, and a total energy of $19 \mathrm{TeV}$ are deposited in the calorimeters. To avoid spoiling the precision measurements this background has to be identified and rejected.

\subsubsection{Rejection of Background Particles}

Due to the small bunch spacing the background from several bunch crossings will pile up over a physics event. To reject the background particles from $\gamma \gamma \rightarrow$ hadron events a time-stamping window of $10 \mathrm{~ns}$ (corresponding to $20 \mathrm{BX}$ ) is required in each sub-detector except for the barrel of the hadronic calorimeter, where a relaxed reconstruction window of $100 \mathrm{~ns}$ is possible. The single hit resolution in each sub-detector has to be about 1 ns [2].

Figure 8 (left) shows a multi-jet event overlaid with the $\gamma \gamma \rightarrow$ hadron background in the $10 \mathrm{~ns}$ reconstruction window of the detectors. The total background energy is $1.2 \mathrm{TeV}$. The rejection of the background particles is based on the average time of the cluster in the calorimeters, which can be precisely estimated from the many hits in an individual cluster. The timing cuts depend on particle type (charged/neutral), the transverse momentum, and the detector region [2]. Three different levels of timing cuts - loose, default and tight - were applied in the standard reconstruction for the conceptual design report. In Figure 8 (right) the tight timing cuts were used to reject the background. The remaining energy from the background particles is only $100 \mathrm{GeV}$, with negligible impact on the underlying hard interaction.

To further reduce the impact of the background hadron-collider inspired jet-clustering algorithms were used. Figure 9 shows the reconstructed energy of $1.1 \mathrm{TeV}$ jets with the $\gamma \gamma \rightarrow$ hadron background overlaid. On the left side the Durham $k_{\mathrm{T}}$-algorithm for electron-positron colliders was used for jet clustering. Here, the distributions depend on the applied timing cuts. The distribution without any timing cuts shows the largest clustered jet energy. On the right side of Figure 9 the hadron-collider $k_{\mathrm{T}}$-algorithm with $R=0.7$ was used for the jet clustering. The difference between the distributions has become much smaller. However, the jets formed from all particles - when the timing cuts were not applied - once again shows a bias towards larger energies [2]. The combination of timing cuts and jet-clustering provide an effective tool to reject the $\gamma \gamma \rightarrow$ hadron background. 

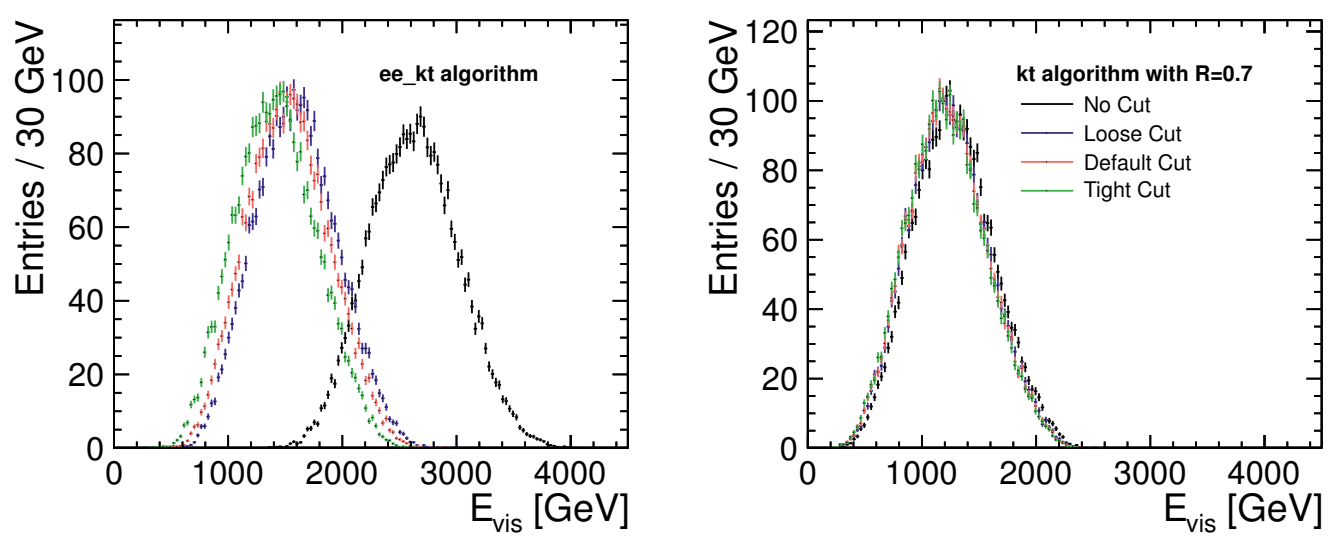

Figure 9. Reconstructed jet energy distributions for different jet clustering algorithms and timing cuts. Left: Durham $k_{\mathrm{T}}$-algorithm, Right: $k_{\mathrm{T}}$-algorithm including beam jets [2].

\subsection{Detector Benchmark Studies and Physics at CLIC}

Detector benchmark studies were performed to evaluate the impact of the CLIC beam structure and background conditions on physics observables at $\sqrt{s}=3 \mathrm{TeV}$. All detector benchmark studies were performed with full GEanT4 simulation, overlaid with $\gamma \gamma \rightarrow$ hadron backgrounds, and fully reconstructed. The sample sizes correspond to $2 \mathrm{ab}^{-1}$ of integrated luminosity, or about four years of nominal operation. The generator values and the results for the benchmark studies described in the following are summarised in Table 2. The benchmark processes consist of rare Standard Model Higgs decays and typical signatures of new physics: missing energy, high momentum leptons, and multi-jet final states. The parameters of the Supersymmetry models (referred to as model I and II) used are described in [10].

\subsubsection{Standard Model Higgs Measurements}

A linear collider allows for the precise measurement of the fundamental properties of the Higgs boson. As the vector-boson fusion cross-section rises with the centre-of-mass energy, more Higgs bosons are produced at $\sqrt{s}=3 \mathrm{TeV}$, which gives access to rare decays, such as the Higgs decaying into two muons. Figure 10 (left) shows the different cross-sections for Higgs production, the rising vectorboson fusion process $(\mathrm{H} v \vee)$ can be clearly seen.

Figure 10 (right) shows the invariant mass of the di-muon system after full simulation and reconstruction. The peak for the Higgs decaying into muons is clearly visible above the background. To not smear out the signal peak an excellent momentum resolution is required. With $2 \mathrm{ab}^{-1}$ a statistical uncertainty of $15.7 \%$ on the measurement of the cross-section times branching-ratio can be achieved [11, 12]. Due to the larger event rate the branching ratio for $\mathrm{c}$ - and b-quarks can be measured with better precision (cf. Table 2).

\subsubsection{Slepton Pair Production}

The reconstruction of high momentum leptons was also studied with the process of slepton pair production. If the slepton decays into its partner lepton and an invisible neutralino the slepton and neu- 
Table 2. Summary table of the CLIC benchmark analyses results. All studies at a centre-of-mass energy of $3 \mathrm{TeV}$ are performed for an integrated luminosity of $2 \mathrm{ab}^{-1}$. The study at $500 \mathrm{GeV}$ assumes an integrated luminosity of $100 \mathrm{fb}^{-1}$ (Table abridged from CDR [2]).

\begin{tabular}{|c|c|c|c|c|c|c|c|}
\hline $\begin{array}{l}\sqrt{s} \\
(\mathrm{TeV})\end{array}$ & Process & Decay mode & $\begin{array}{l}\text { SUSY } \\
\text { model }\end{array}$ & Observable & Unit & $\begin{array}{l}\text { Gene- } \\
\text { rator } \\
\text { value }\end{array}$ & $\begin{array}{l}\text { Stat. } \\
\text { uncert- } \\
\text { ainty }\end{array}$ \\
\hline \multirow{3}{*}{3.0} & \multirow{3}{*}{$\begin{array}{l}\text { Light Higgs } \\
\text { production }\end{array}$} & $\mathrm{h} \rightarrow \mathrm{b} \overline{\mathrm{b}}$ & & \multirow{3}{*}{$\begin{array}{l}\sigma \\
\times \text { Bran- } \\
\text { ching ratio }\end{array}$} & \multirow{3}{*}{$\mathrm{fb}$} & 285 & $0.22 \%$ \\
\hline & & $\mathrm{h} \rightarrow \mathrm{c} \overline{\mathrm{c}}$ & & & & 13 & $3.2 \%$ \\
\hline & & $\mathrm{h} \rightarrow \mu^{+} \mu^{-}$ & & & & 0.12 & $15.7 \%$ \\
\hline \multirow{8}{*}{3.0} & \multirow{8}{*}{$\begin{array}{l}\text { Heavy Higgs } \\
\text { production }\end{array}$} & \multirow{4}{*}{$\mathrm{HA} \rightarrow \mathrm{b} \bar{b} \mathrm{~b} \bar{b}$} & \multirow{2}{*}{ I } & Mass & $\mathrm{GeV}$ & 902.4 & $0.3 \%$ \\
\hline & & & & Width & $\mathrm{GeV}$ & & $31 \%$ \\
\hline & & & \multirow{2}{*}{ II } & Mass & $\mathrm{GeV}$ & 742.0 & $0.2 \%$ \\
\hline & & & & Width & $\mathrm{GeV}$ & & $17 \%$ \\
\hline & & \multirow{4}{*}{$\mathrm{H}^{+} \mathrm{H}^{-} \rightarrow \mathrm{t} \bar{b} \mathrm{~b} \overline{\mathrm{t}}$} & \multirow{2}{*}{ I } & Mass & $\mathrm{GeV}$ & 906.3 & $0.3 \%$ \\
\hline & & & & Width & $\mathrm{GeV}$ & & $27 \%$ \\
\hline & & & \multirow{2}{*}{ II } & Mass & $\mathrm{GeV}$ & 747.6 & $0.3 \%$ \\
\hline & & & & Width & $\mathrm{GeV}$ & & $23 \%$ \\
\hline \multirow{10}{*}{3.0} & \multirow{10}{*}{$\begin{array}{l}\text { Slepton } \\
\text { production }\end{array}$} & & \multirow{10}{*}{ II } & $\sigma$ & $\mathrm{fb}$ & 0.72 & $2.8 \%$ \\
\hline & & $\tilde{\mu}_{\mathrm{R}}^{+} \tilde{\mu}_{\mathrm{R}}^{-} \rightarrow \mu^{+} \mu^{-} \tilde{\chi}_{1}^{0} \tilde{\chi}_{1}^{0}$ & & $\tilde{\ell}$ mass & $\mathrm{GeV}$ & 1010.8 & $0.6 \%$ \\
\hline & & & & $\tilde{\chi}_{1}^{0}$ mass & $\mathrm{GeV}$ & 340.3 & $1.9 \%$ \\
\hline & & & & $\sigma$ & $\mathrm{fb}$ & 6.05 & $0.8 \%$ \\
\hline & & $\widetilde{\mathrm{e}}_{\mathrm{R}}^{+} \widetilde{\mathrm{e}}_{\mathrm{R}}^{-} \rightarrow \mathrm{e}^{+} \mathrm{e}^{-} \tilde{\chi}_{1}^{0} \tilde{\chi}_{1}^{0}$ & & $\tilde{\ell}$ mass & $\mathrm{GeV}$ & 1010.8 & $0.3 \%$ \\
\hline & & & & $\tilde{\chi}_{1}^{0}$ mass & $\mathrm{GeV}$ & 340.3 & $1.0 \%$ \\
\hline & & $\begin{array}{l}\widetilde{\mathrm{e}}_{\mathrm{L}}^{+} \widetilde{\mathrm{e}}_{\mathrm{L}}^{-} \rightarrow \tilde{\chi}_{1}^{0} \tilde{\chi}_{1}^{0} \mathrm{e}^{+} \mathrm{e}^{-} \mathrm{hh} \\
\widetilde{\mathrm{e}}_{\mathrm{L}}^{+} \widetilde{\mathrm{e}}_{\mathrm{L}}^{-} \rightarrow \tilde{\chi}_{1}^{0} \tilde{\chi}_{1}^{0} \mathrm{e}^{+} \mathrm{e}^{-} \mathrm{Z}^{0} \mathrm{Z}^{0}\end{array}$ & & $\sigma$ & $\mathrm{fb}$ & 3.07 & $7.2 \%$ \\
\hline & & \multirow{3}{*}{$\widetilde{v}_{\mathrm{e}} \widetilde{v}_{\mathrm{e}} \rightarrow \tilde{\chi}_{1}^{0} \tilde{\chi}_{1}^{0} \mathrm{e}^{+} \mathrm{e}^{-} \mathrm{W}^{+} \mathrm{W}^{-}$} & & $\sigma$ & $\mathrm{fb}$ & 13.74 & $2.4 \%$ \\
\hline & & & & $\tilde{\ell}$ mass & $\mathrm{GeV}$ & 1097.2 & $0.4 \%$ \\
\hline & & & & $\tilde{\chi}_{1}^{ \pm}$mass & $\mathrm{GeV}$ & 643.2 & $0.6 \%$ \\
\hline \multirow{4}{*}{3.0} & \multirow{4}{*}{$\begin{array}{l}\text { Chargino } \\
\text { and } \\
\text { neutralino } \\
\text { production }\end{array}$} & \multirow[t]{2}{*}{$\tilde{\chi}_{1}^{+} \tilde{\chi}_{1}^{-} \rightarrow \tilde{\chi}_{1}^{0} \tilde{\chi}_{1}^{0} \mathrm{~W}^{+} \mathrm{W}^{-}$} & \multirow{4}{*}{ II } & $\tilde{\chi}_{1}^{ \pm}$mass & $\mathrm{GeV}$ & 643.2 & $1.1 \%$ \\
\hline & & & & $\sigma$ & $\mathrm{fb}$ & 10.6 & $2.4 \%$ \\
\hline & & \multirow[t]{2}{*}{$\tilde{\chi}_{2}^{0} \tilde{\chi}_{2}^{0} \rightarrow \mathrm{h}^{0} / \mathrm{Z}^{0} \mathrm{~h}^{0} / \mathrm{Z}^{0} \tilde{\chi}_{1}^{0} \tilde{\chi}_{1}^{0}$} & & $\tilde{\chi}_{2}^{0}$ mass & $\mathrm{GeV}$ & 643.1 & $1.5 \%$ \\
\hline & & & & $\sigma$ & $\mathrm{fb}$ & 3.3 & $3.2 \%$ \\
\hline \multirow{4}{*}{0.5} & \multirow{4}{*}{$\overline{\mathrm{t}}$ production } & \multirow{2}{*}{$\bar{t} \bar{t} \rightarrow(q \bar{q} b)(q \bar{q} b)$} & & Mass & $\mathrm{GeV}$ & 174 & $0.046 \%$ \\
\hline & & & & Width & $\mathrm{GeV}$ & 1.37 & $16 \%$ \\
\hline & & \multirow{2}{*}{$\begin{array}{l}\mathrm{t} \overline{\mathrm{t}} \rightarrow(\mathrm{q} \overline{\mathrm{q}} \mathrm{b})(\ell v \mathrm{~b}) \\
\ell=\mathrm{e}, \mu\end{array}$} & & Mass & $\mathrm{GeV}$ & 174 & $0.052 \%$ \\
\hline & & & & Width & $\mathrm{GeV}$ & 1.37 & $18 \%$ \\
\hline
\end{tabular}



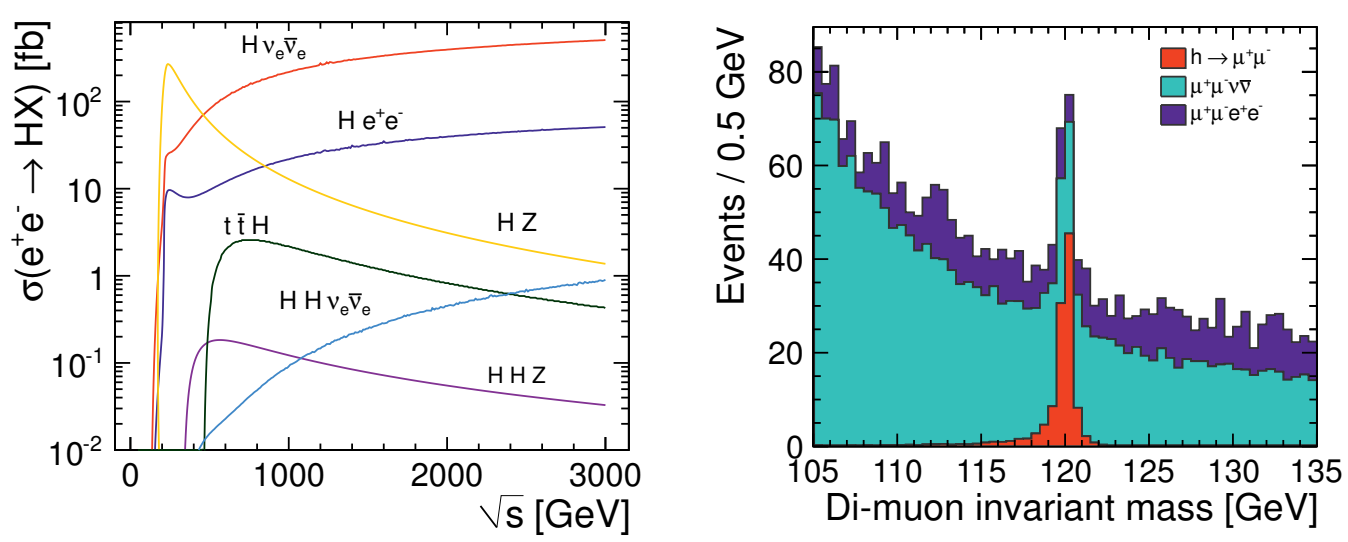

Figure 10. Left: Higgs production cross-section for $m_{\mathrm{h}}=120 \mathrm{GeV}$. Right: Di-muon invariant mass spectrum, the peak at the Higgs mass is clearly visible above the background [2].

tralino mass can be extracted from the endpoints of the lepton energy spectrum [5]. Figure 11 shows the distribution of the reconstructed energy of muons after background subtraction. The shape differs from the expected rectangular one because of the luminosity spectrum and finite momentum resolution. The figure also contains a fit which takes into account these imperfections. The pair production of scalar electrons and scalar neutrinos was also studied. The masses of these sleptons and the neutralino can be measured with a statistical uncertainty of a few GeV (cf. Table 2).

\subsubsection{Gaugino Pair Production}

The final state of the different chargino and neutralino pair production processes is always four jets (from different boson pairs) and missing energy (in the neutralino $\widetilde{\chi}_{1}^{0}$ ):

$$
\begin{aligned}
& \mathrm{e}^{+} \mathrm{e}^{-} \rightarrow \widetilde{\chi}_{1}^{+} \widetilde{\chi}_{1}^{-} \rightarrow \widetilde{\chi}_{1}^{0} \tilde{\chi}_{1}^{0} \mathrm{~W}^{+} \mathrm{W}^{-} \\
& \mathrm{e}^{+} \mathrm{e}^{-} \rightarrow \widetilde{\chi}_{2}^{0} \widetilde{\chi}_{2}^{0} \rightarrow \widetilde{\chi}_{1}^{0} \widetilde{\chi}_{1}^{0} \mathrm{hh} \\
& \mathrm{e}^{+} \mathrm{e}^{-} \rightarrow \widetilde{\chi}_{2}^{0} \tilde{\chi}_{2}^{0} \rightarrow \widetilde{\chi}_{1}^{0} \widetilde{\chi}_{1}^{0} \mathrm{Zh} .
\end{aligned}
$$

To separate the different processes - and measure the mass of the respective gaugino - the jet masses have to be reconstructed, so that the original bosons can be identified. Figure 12 shows the reconstructed di-jet mass for the three different Gaugino pair production processes; the peak of each process is well separated from the others. This demonstrates that the particle flow reconstruction of boosted bosons performs well in the CLIC environment [13].

\subsubsection{Heavy Higgs Bosons}

Heavy Higgs bosons are a feature of many extensions of the Standard Model. In the chosen model (see [10]) the heavy Higgs bosons mainly decay into multiple b- and t- quarks. This process therefore tests the reconstruction of high-mass, multi-jet final states and requires a high b-tagging efficiency. Figure 13 shows the reconstructed invariant di-jet mass of the neutral and charged Higgs pair production processes. A statistical resolution on the mass of less than $0.3 \%$ is achieved [2]. 


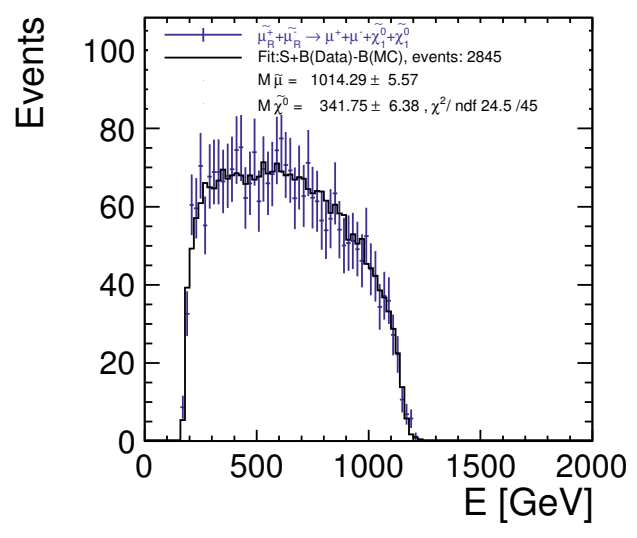

Figure 11. Reconstructed muon energy spectrum for the smuon pair production process after background subtraction.

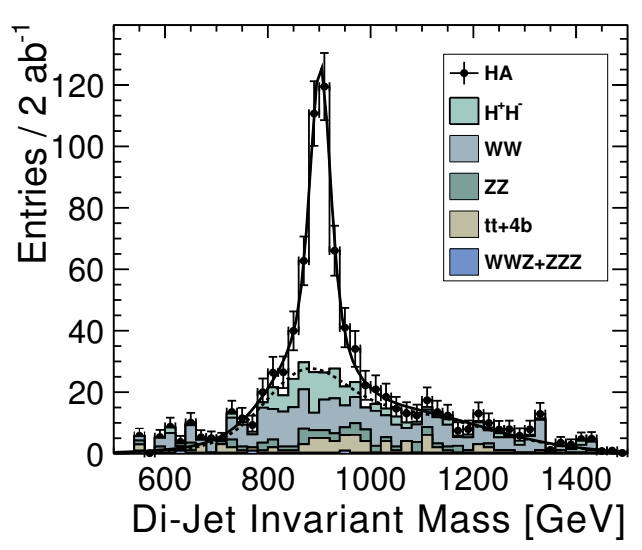

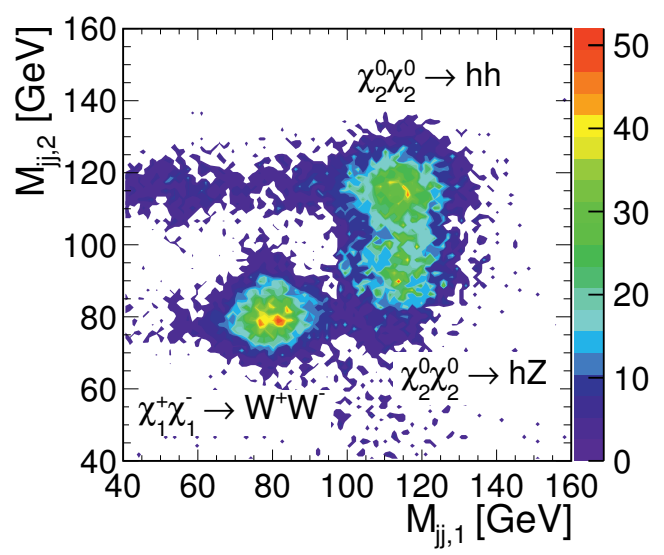

Figure 12. Scatter plot showing the reconstructed di-jet invariant masses of $\mathrm{W}, \mathrm{Z}$ and $\mathrm{h}$ candidates in simulated $\mathrm{e}^{+} \mathrm{e}^{-} \rightarrow \tilde{\chi}_{1}^{+} \tilde{\chi}_{1}^{-}$and $\mathrm{e}^{+} \mathrm{e}^{-} \rightarrow \tilde{\chi}_{2}^{0} \tilde{\chi}_{2}^{0}$ signal events including $\gamma \gamma \rightarrow$ hadrons background. The peaks corresponding to the individual chargino and neutralino decays are indicated. The event samples were scaled to have a similar number of events for each channel [2].

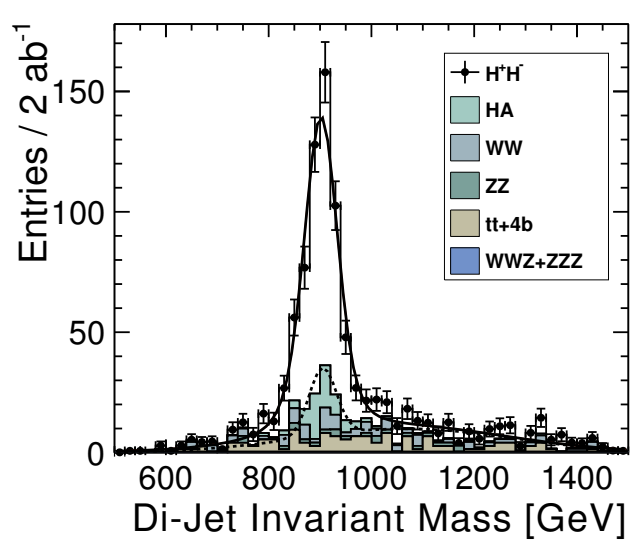

Figure 13. Di-jet invariant mass distributions for the $\mathrm{b} \overline{\mathrm{b}} \mathrm{b} \overline{\mathrm{b}}$ (left) and thb $\overline{\mathrm{b}}$ (right) final states. The distributions for the $\mathrm{e}^{+} \mathrm{e}^{-} \rightarrow \mathrm{HA}$ and $\mathrm{e}^{+} \mathrm{e}^{-} \rightarrow \mathrm{H}^{+} \mathrm{H}^{-}$processes and for the individual backgrounds are shown separately [2]. 

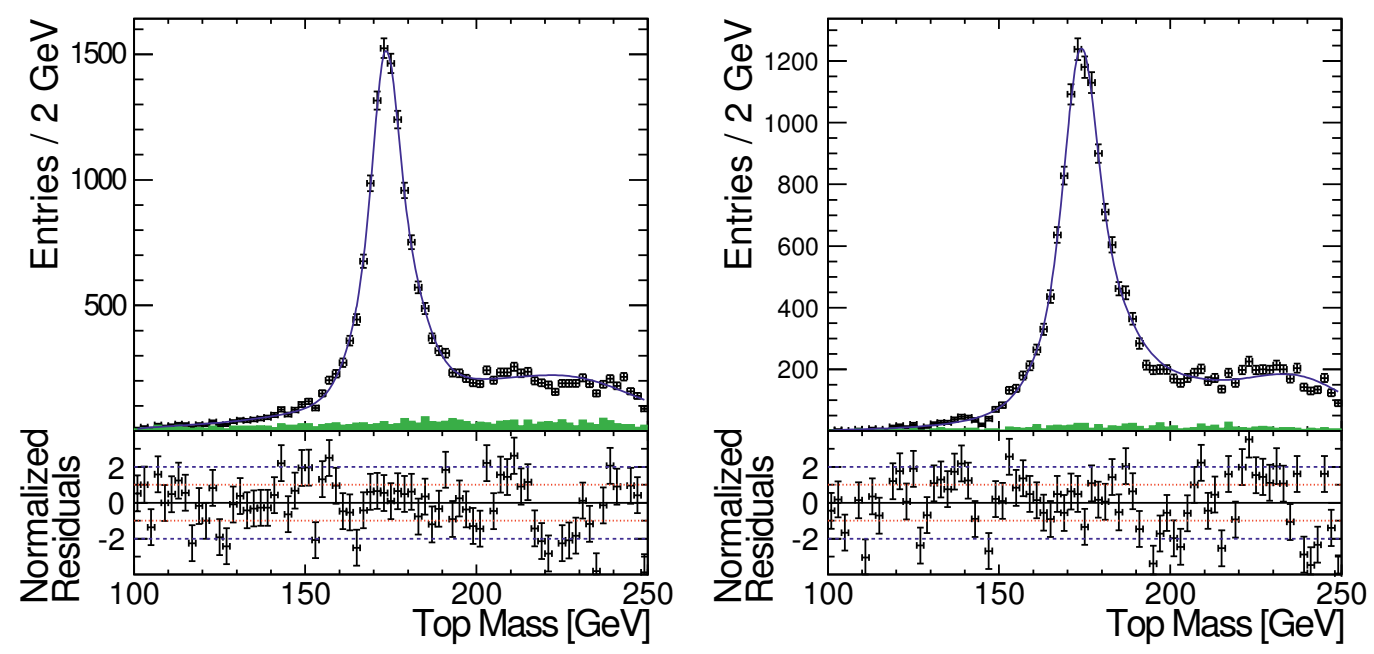

Figure 14. Mass distribution for 6-jet events (left) and 4-jet events (right). Points with error bars show simulated data classified as signal events. The green histogram illustrates the contribution of non-tt background to the distribution. The blue line shows the fit of the top mass distribution [2].

\subsection{5 tī at $500 \mathrm{GeV}$}

The study of the top-quark would be a core part of a first CLIC stage [3]. The top-pair production was simulated at a centre-of-mass energy of $500 \mathrm{GeV}$ and studied with a data sample corresponding to an integrated luminosity of $100 \mathrm{fb}^{-1}$. Figure 14 shows the distributions of the reconstructed top-masses for the fully hadronic channel (both W-bosons decaying to four jets, and the semi-leptonic channel (one W-boson decaying to lepton and neutrino). In the fully hadronic channel a statistical uncertainty of about $80 \mathrm{MeV}$ on the mass of the top-quark can be reached [14].

\subsubsection{Summary}

Precision measurements for a wide variety of new physics signatures can be performed at CLIC, despite the challenging background and beam conditions at $3 \mathrm{TeV}$. Rare Higgs decays profit from the larger cross-section at higher centre-of-mass energies. CLIC can also be used as a discovery machine complementary to the LHC. Table 3 lists the physics reach for hadron and electron-positron colliders with different centre-of-mass energies and integrated luminosities. A high energy electron-positron collider has advantages when precision measurements are required to find new physics. Due to the cleaner conditions electro-weak particles can be discovered in the full energy range of the collider up to a mass of $m_{x}=\sqrt{s} / 2$. CLIC can also be sensitive to new physics far beyond its direct energy reach, for example, evidence for a $Z^{\prime}$ with Standard Model couplings could be found up to a $Z^{\prime}$ mass of $20 \mathrm{TeV}$.

\subsection{Physics and Detectors: Future Plans}

For the next project phase the physics performance of CLIC at various energy stages will be studied, keeping a close eye on the results from the LHC. The detector design will be further refined and optimised. 
Table 3. Physics reach for different colliders. TGC is short for Triple Gauge Coupling, and " $\mu$ contact scale" is short for LL $\mu$ contact interaction scale $\Lambda$ with $g=1[2,15]$.

\begin{tabular}{lcccc}
\hline & $\begin{array}{c}\text { LHC14 } \\
100 \mathrm{fb}^{-1}\end{array}$ & $\begin{array}{c}\text { SLHC14 } \\
1 \mathrm{ab}^{-1}\end{array}$ & $\begin{array}{c}\text { LC800 } \\
500 \mathrm{fb}^{-1}\end{array}$ & $\begin{array}{c}\text { CLIC3 } \\
1 \mathrm{ab}^{-1}\end{array}$ \\
\hline squarks $[\mathrm{TeV}]$ & 2.5 & 3 & 0.4 & 1.5 \\
sleptons $[\mathrm{TeV}]$ & 0.3 & & 0.4 & 1.5 \\
$\mathrm{Z}_{\text {(SM couplings) }}[\mathrm{TeV}]$ & 5 & 7 & 8 & 20 \\
2 extra dims $M_{D}[\mathrm{TeV}]$ & 9 & 12 & $5-8.5$ & $20-30$ \\
$\mu$ contact scale $[\mathrm{TeV}]$ & 15 & & 20 & 60 \\
Higgs composite scale $[\mathrm{TeV}]$ & $5-7$ & $9-12$ & 45 & 60 \\
TGC (95\%) (ay coupling) & 0.001 & 0.0006 & 0.0004 & 0.0001 \\
\hline
\end{tabular}

There are many technological challenges for the sub-detectors, which require $R \& D$. The vertex detector has to be ultra-thin, small-pixeled and fast. The main tracking detectors need an integrated design. The electronics for the sensors require fast-timing, while offering a low power-consumption and the ability for power-pulsing. The highly granular calorimeters require compact active layers and $1 \mathrm{~ns}$ hit time resolution. At the same time the engineering challenges for the support, stability, alignment, and cooling have to be met. These topics are part of an ongoing R\&D effort for the postCDR project phase of 2012-2016 [2].

\section{Summary}

The conceptual design reports for the Compact Linear Collider show good results, proving the feasibility of the accelerator and detector technologies. The drive-beam generation, and its deceleration in power extraction structures have been shown in CTF3. The RF power provided by the drive beam was used to accelerate a second beam with a gradient of $106 \mathrm{MV} / \mathrm{m}$. The break-down rate of the accelerator cavities and PETS was reduced to the required level. Despite the challenging background conditions at CLIC precision physics measurements can be performed. Rare Higgs decays can be studied and extensions of the Standard Model can be explored with great precision. CLIC therefore offers a promising possibility to study fundamental physics complementary to the LHC.

\section{References}

[1] M. Aicheler, P. Burrows, M. Draper, T. Garvey, P. Lebrun, K. Peach, N. Phinney, H. Schmickler, D. Schulte, N. Toge, eds., A Multi-TeV Linear Collider based on CLIC Technology: CLIC Conceptual Design Report (CERN, 2012), JAI-2012-001, KEK Report 2012-1, PSI-12-01, SLACR-985

[2] L. Linssen, A. Miyamoto, M. Stanitzki, H. Weerts, eds., Physics and Detectors at CLIC: CLIC Conceptual Design Report (CERN, 2012), ANL-HEP-TR-12-01, CERN-2012-003, DESY 12008, KEK Report 2011-7

[3] P. Lebrun, L. Linssen, A. Lucaci-Timoce, D. Schulte, F. Simon, S. Stapnes, N. Toge, H. Weerts, J.D. Wells, eds., The CLIC Programme: towards a staged $e^{+} e^{-}$Linear Collider exploring the Terascale (CERN, 2012), ANL-HEP-TR-12-51, KEK Report 2012-2, MPP-2012-115

[4] L.R. Evans, P. Bryant, J. Instrum. 3, S08001. 164 p (2008) 
[5] M. Battaglia, J.J. Blaising, J. Marshall, J. Nardulli, M.A. Thomson, E. van der Kraaij, Physics performances for scalar electrons, scalar muons and scalar neutrinos searches at CLIC, CERNLCD-Note-2011-018 (2011)

[6] A. Münnich, A. Sailer, The CLIC_ILD_CDR Geometry for the CDR Monte Carlo Mass Production, CERN-LCD-Note-2011-002 (2011)

[7] C. Grefe, A. Münnich, The CLIC_SiD_CDR Geometry for the CDR Monte Carlo Mass Production, CERN-LCD-Note-2011-009 (2011)

[8] P. Chen, An Introduction to Beamstrahlung and Disruption, in Frontiers of Particle Beams, edited by M. Month, S. Turner (1988), Vol. 296 of Lecture Notes in Physics, Berlin Springer Verlag, pp. 495-532

[9] P. Chen, V. Telnov, Phys. Rev. Lett. 63, 1796 (1989)

[10] M.A. Thomson, M. Battaglia, J. Ellis, G. Giudice, A. Lucaci-Timoce, F. Teubert, J. Wells, J.J. Blaising, S. Martin, The physics benchmark processes for the detector performance studies of the CLIC CDR, CERN-LCD-Note-2011-016 (2011)

[11] C. Grefe, Light Higgs decay into muons in the CLIC_SiD CDR detector, CERN-LCD-Note2011-035 (2011)

[12] C. Grefe, T. Lastovicka, J. Strube, accepted by Eur. Phys. J. C (2012), hep-ex/1208 . 2890

[13] T. Barklow, A. Münnich, P. Roloff, Measurement of chargino and neutralino pair production at CLIC, CERN-LCD-Note-2011-037 (2011)

[14] K. Seidel, S. Poss, F. Simon, Top quark pair production at a $500 \mathrm{GeV}$ CLIC collider, CERNLCD-Note-2011-026 (2011)

[15] A. de Roeck, J.R. Ellis, F. Gianotti, Physics motivations for future cern accelerators. oai:cds.cern.ch:529240, hep-ex/0112004. CERN-TH-2001-023 (2001) 